Review Article

\title{
Targeting the Mitochondrial Permeability Transition Pore to Prevent Age-Associated Cell Damage and Neurodegeneration
}

\author{
Andrew C. Kent, ${ }^{1,2}$ Khairat Bahgat Youssef El Baradie, ${ }^{1,3}$ and Mark W. Hamrick $\mathbb{D}^{1}$ \\ ${ }^{1}$ Medical College of Georgia, Augusta University, Augusta, GA 30912, USA \\ ${ }^{2}$ University of Georgia, Athens, GA, USA \\ ${ }^{3}$ Faculty of Science, Tanta University, Tanta, Egypt
}

Correspondence should be addressed to Mark W. Hamrick; mhamrick@augusta.edu

Received 1 December 2020; Revised 19 January 2021; Accepted 21 January 2021; Published 28 January 2021

Academic Editor: Fabrizio Biundo

Copyright $\odot 2021$ Andrew C. Kent et al. This is an open access article distributed under the Creative Commons Attribution License, which permits unrestricted use, distribution, and reproduction in any medium, provided the original work is properly cited.

\begin{abstract}
The aging process is associated with significant alterations in mitochondrial function. These changes in mitochondrial function are thought to involve increased production of reactive oxygen species (ROS), which over time contribute to cell death, senescence, tissue degeneration, and impaired tissue repair. The mitochondrial permeability transition pore (mPTP) is likely to play a critical role in these processes, as increased ROS activates mPTP opening, which further increases ROS production. Injury and inflammation are also thought to increase mPTP opening, and chronic, low-grade inflammation is a hallmark of aging. Nicotinamide adenine dinucleotide (NAD+) can suppress the frequency and duration of mPTP opening; however, NAD+ levels are known to decline with age, further stimulating MPTP opening and increasing ROS release. Research on neurodegenerative diseases, particularly on Parkinson's disease (PD) and Alzheimer's disease (AD), has uncovered significant findings regarding mPTP openings and aging. Parkinson's disease is associated with a reduction in mitochondrial complex I activity and increased oxidative damage of DNA, both of which are linked to MPTP opening and subsequent ROS release. Similarly, AD is associated with increased mPTP openings, as evidenced by amyloid-beta $(\mathrm{A} \beta)$ interaction with the pore regulator cyclophilin $\mathrm{D}(\mathrm{CypD})$. Targeted therapies that can reduce the frequency and duration of $\mathrm{mPTP}$ opening may therefore have the potential to prevent age-related declines in cell and tissue function in various systems including the central nervous system.
\end{abstract}

\section{Introduction}

The number of older adults is growing worldwide. As a result, the incidence of age-associated diseases including $\mathrm{AD}$, osteoporosis, sarcopenia, and osteoarthritis is also increasing. This increase in age-related disorders has a significant, negative impact on the quality of life for patients and their families and also places a substantial burden on healthcare systems. A better understanding of the cellular and molecular mechanisms underlying aging is central to the successful development and clinical translation of novel therapies and prevention strategies. Recent work has demonstrated that changes in MPTP function may contribute directly to cellular dysfunction with aging [1-3]. These changes include increases in ROS production, induction of cellular senescence (particularly in aging stem cells), and activation of the inflammasome, the latter contributing directly to the chronic state of inflammation often referred to as "inflammaging" [1-3]. MPTP dysfunction has been cited as a key factor in neurodegenerative pathologies through its role in collapsing mitochondrial membrane potential, repressing mitochondrial respiratory function, releasing mitochondrial $\mathrm{Ca}^{2+}$ and cytochrome $\mathrm{c}$, and enhancing ROS generation [47]. Thus, the MPTP has received increased attention as a potential therapeutic target.

The relationship between the $\mathrm{mPTP}$ and the generation of mitochondrial reactive oxygen species (mROS) has attracted significant interest within the context of aging and age-related tissue degeneration [8]. Recently, it was found that mROS can stimulate the opening of the $\mathrm{mPTP}$, which can lead to further mROS production and release [9]. This positive feedback mechanism ultimately leads to an excessive amount of ROS accumulation. ROS accumulation in turn damages nuclear DNA, activates proapoptotic signaling 
pathways, and drives cellular aging [10-12]. On the other hand, ROS can in some cases activate protective pathways, decrease stress on the mitochondria, and increase lifespan $[1,11]$. It is currently thought that the MPTP plays an important role in integrating the effects of mROS and hence may play a vital role in the aging process [8]. In this review, we discuss the various mechanisms inducing activation of the mPTP and the age-associated cell damage seen as a byproduct of MPTP activation. Furthermore, we discuss potential therapies that target the $\mathrm{MPTP}$ and may therefore inhibit the effects of aging and injury.

1.1. Structure and Formation of the MPTP. Various structural components of the MPTP are implicated in permeability transition (PT); however, the overall structure of the mPTP is still not completely understood. It was previously thought that the pore consisted of several components including a voltage-dependent ion channel (VDAC), an adenine nucleotide transporter (ANT), and a peripheral benzodiazepine receptor $[13,14]$. These elements are described to perform specific roles: VDAC is associated with the benzodiazepine receptor and regulates the extramitochondrial transfer of cholesterol to the intermembrane space whereas ANT permits the inflow of phosphorylated and nonphosphorylated derivatives of adenine nucleotides [15]. Except for ANT, which is thought to act as a potential regulatory molecule, recent genetic experiments have ruled out the aforementioned elements as components of the mPTP [16]. Thus, we present here the most recent models regarding $\mathrm{MPTP}$ composition with the understanding that these may be revised in the near future.

Recent models of pore composition posit that the $\mathrm{F}_{1} \mathrm{~F}_{0}$ (F)-ATP synthase is the main component of the pore and that the regulatory molecule CypD is a protein modulator of the mPTP [17]. In this model, the mPTP originates from a conformational change occurring on the $\mathrm{F}_{1} \mathrm{~F}_{0}(\mathrm{~F})$-ATP synthase after $\mathrm{Ca}^{2+}$ binding, possibly by replacing $\mathrm{Mg}^{2+}$ at the catalytic site [18]. Whether the dimeric form or the monomeric form of $\mathrm{F}_{1} \mathrm{~F}_{0}(\mathrm{~F})$-ATP synthase is necessary to increase $\mathrm{PT}$ is still of great debate $[19,20]$. Nevertheless, $\mathrm{F}_{1} \mathrm{~F}_{0}(\mathrm{~F})$-ATP synthase's status as a pore component is supported by genetic manipulation of $F_{1} F_{0}(F)$-ATP synthase $[20,21]$, by electrophysiological measurements [20,22-24], and by mutagenesis of specific residues of $\mathrm{F}_{1} \mathrm{~F}_{0}(\mathrm{~F})$-ATP synthase [18, 25-27]. On the other hand, Walker and colleagues have proposed that the $\mathrm{F}_{1} \mathrm{~F}_{0}(\mathrm{~F})$-ATP synthase is not an essential component of the pore $[28,29]$. Their hypothesis is based on the observation that, even after ablating subunits $b$ and OSCP of $\mathrm{F}_{1} \mathrm{~F}_{0}$ (F)-ATP synthase, mitochondrial PT still occurred [29]. Matrix swelling was used to determine PT because longlasting $\mathrm{mPTP}$ opening in vitro is followed by solute diffusion with matrix swelling [30].

Questions have, however, been raised regarding these findings. Bernardi [17] in particular noted the absence of replicates and calibration with pore-forming agents like alamethicin may complicate interpretation of the data. The effects on respiration following $\mathrm{F}_{1} \mathrm{~F}_{0}$ (F)-ATP synthase knockout raise additional questions. Respiratory activity was dramatically decreased to between 10 and $20 \%$ of the rate observed in wild-type cells after $\mathrm{F}_{1} \mathrm{~F}_{0}(\mathrm{~F})$-ATP synthase knockout [29]. The driving force in respiring mitochondria for $\mathrm{Ca}^{2+}$ accumulation is the inside-negative membrane potential generated by respiration [31, 32]. Furthermore, $\mathrm{Ca}^{2+}$ uptake is charge-compensated by increased $\mathrm{H}+$ pumping by the respiratory chain [17]. Thus, it is important to note that the maximal rate of $\mathrm{Ca}^{2+}$ uptake is limited by the maximal rate of $\mathrm{H}+$ pumping by the respiratory chain [33]. When extramitochondrial $\mathrm{Ca}^{2+}$ levels exceed $2 \mu \mathrm{M}$, the latter becomes rate-limiting [34]. He et al. [28] used $10 \mu \mathrm{M}$ pulses of $\mathrm{Ca}^{2+}$ to induce PT; therefore, $\mathrm{Ca}^{2+}$ uptake by mitochondria lacking subunits $c, b$, and OSCP should have been significantly lower and not identical to wild-type mitochondria [17]. This raises questions about the $\mathrm{Ca}^{2+}$ retention capacity, a measurement used by $\mathrm{He}$ et al. [28] to determine mPTP opening. It is possible that respiratory inhibition due to absence of certain subunits may not be constant over time. Potential mechanisms may exist that restore the expression of $\mathrm{F}_{1} \mathrm{~F}_{0}(\mathrm{~F})$-ATP synthase and by consequence the respiratory chain. When considering the above findings, $\mathrm{F}_{1} \mathrm{~F}_{0}(\mathrm{~F})$-ATP synthase cannot necessarily be ruled out as a pore component.

The most compelling experiments supporting $\mathrm{F}_{1} \mathrm{~F}_{0}(\mathrm{~F})$ ATP synthase as a pore component focus on the mutagenesis of specific residues of F-ATP synthase. Specifically, it was found that matrix $\mathrm{H}+$ leads to inhibition of $\mathrm{mPTP}$ and complete channel block at pH $6.5[25,35]$. It was found that the mPTP block is mediated by reversible protonation of matrix-accessible His residues [35]. Recently, H112 of the OSCP subunit has been implicated as the unique His responsible for the PTP block by $\mathrm{H}+$ [25]. Although these findings are intriguing with respect to $\mathrm{MPTP}$ activity, they serve a dual purpose in also supporting OSCP and by consequence $\mathrm{F}_{1} \mathrm{~F}_{0}$ (F)-ATP synthase as potential components of the mPTP. Further controversial components include ANT, which may serve a regulatory role by binding $\mathrm{CypD}$ and reconstituting into proteoliposomes, producing $\mathrm{Ca}^{2+}$-activated pores similar to the mPTP $[36,37]$ and the mitochondrial phosphate carrier PiC $[38,39]$. Thus, potential constituents of the MPTP include ANT, PiC, and $\mathrm{F}_{1} \mathrm{~F}_{0}(\mathrm{~F})$-ATP synthase (Figure 1). Although its role is controversial, we emphasize the potential role of $\mathrm{F}_{1} \mathrm{~F}_{0}(\mathrm{~F})$-ATP synthase in mitochondrial permeability.

$\mathrm{F}_{1} \mathrm{~F}_{0}(\mathrm{~F})$-ATP synthase's various interactions with molecules such as CypD result in increased mitochondrial permeability. The specific subunits of $\mathrm{F}_{1} \mathrm{~F}_{0}(\mathrm{~F})$-ATP synthase have been studied in relation to their interaction with regulatory molecules such as CypD. It is thought that mammalian $\mathrm{F}_{1} \mathrm{~F}_{0}(\mathrm{~F})$-ATP synthase is a protein complex composed of the following: an $\mathrm{F}_{1}$ region composed of $(\alpha \beta) 3, \gamma, \delta$, and $\varepsilon$ subunits, which protrudes in the matrix and synthesizes/hydrolyzes ATP; an $\mathrm{F}_{0}$ sector, formed from $a$ subunit, the c8ring, two membrane-inserted $\alpha$-helices of b subunit, and supernumeraries subunits e, f, g, k, A6L, diabetes-associated protein in insulin-sensitive tissue (DAPIT) and $6.8 \mathrm{kDa}$ proteolipid, which allows $\mathrm{H}+$ flow across the IMM; the central stalk complex; and the peripheral stalk subcomplex composed of the following: oligomycin sensitivity conferral protein (OSCP), d, F6, and the extrinsic $\alpha$-helices of A6L and b subunits (Figure 1) [40]. 


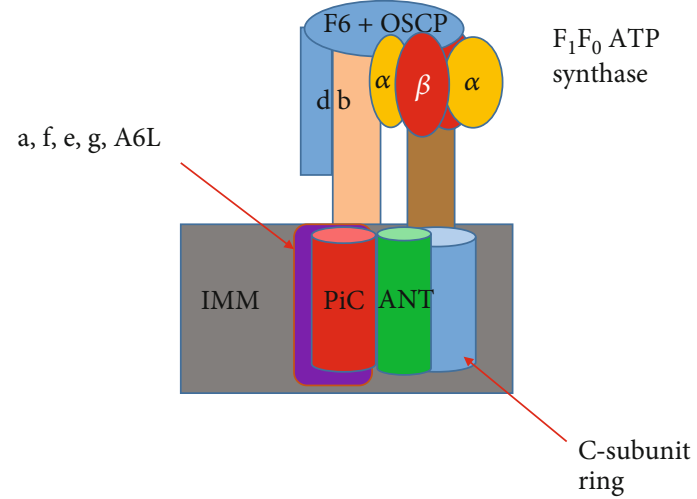

Figure 1: Prevailing model concerning the makeup of the mPTP as formed by the following potential components: mammalian $\mathrm{F}_{1} \mathrm{~F}_{0}$ (F)-ATP synthase, Adenine nucleotide translocator (ANT), and mitochondrial phosphate carrier $(\mathrm{PiC})$. The figure is redrawn and adapted based on reference [138]. Although ANT and PiC remain controversial potential components of the MPTP, they are shown as both red and green components overlaying the inner mitochondrial membrane (IMM). Similarly, although $\mathrm{F}_{1} \mathrm{~F}_{0}(\mathrm{~F})$ ATP synthase is a controversial component, it is labeled as follows. Subunits of the $\mathrm{F}_{0}$ component labeled in purple include a, $\mathrm{e}, \mathrm{f}, \mathrm{g}$, and A6L. $\mathrm{F}_{1}$ components include $\alpha$ and $\beta$ subunits labeled in yellow and red, respectively. The $C$ ring subunit is labeled in blue represented by a cylinder. The $F_{1}$ peripheral stalk is composed of the subunits b, d, F6, and oligomycin sensitivity conferring protein (OSCP) labeled represented by a peach rectangle, a blue rectangle, and a blue circle, respectively. The mPTP is the point at which ROS, $\mathrm{Ca}^{2+}$, and other molecules can escape from the matrix of the mitochondria.

OSCP and CypD interact to promote the opening of the mPTP, and further, mPTP opening is increased with aging and oxidative stress [41-43]. Oxidative stress induces the translocation of the tumor suppressor p53 to the mitochondrial matrix where it interacts with CypD to aid in the formation of the MPTP [44]. Like the oxidative stress-induced formation of the mPTP, $\mathrm{Ca}^{2+}$ can also induce formation of the MPTP. It has been found that soluble matrix peptidylprolyl isomerase F cyclophilin D (PPIF) is involved in the $\mathrm{Ca}^{2+}$ induced opening of the MPTP [15]. The interaction between the aforementioned molecules, oxidative stress, and $\mathrm{Ca}^{2+}$ overloading can change significantly across the lifespan.

1.2. Role of the MPTP in Cellular Aging. A range of studies indicates that MPTP activation is altered with age in a variety of cell and tissue types. These include permeabilized myofibrils in humans [45], myocytes in rats [46], and osteocytes in mice [47]. It should be noted, before discussing the various effects of aging on mPTP activation, that $\mathrm{Ca}^{2+}$ is a wellestablished activator of the mPTP [48]. Specifically, concerning the permeabilized myofibrils in humans, Gouspillou et al. [28] found that $\mathrm{Ca}^{2+}$ retention and time to mPTP opening were significantly decreased in skeletal muscle of older active men [45]. Decreased $\mathrm{Ca}^{2+}$ retention is indicative of mPTP openings [45]. It was also found that the mPTP of older, active men maintains an increased sensitivity to $\mathrm{Ca}^{2+}$, further supporting the idea that increased MPTP activation is a byproduct of aging. These results are further reinforced by work showing that oxidative damage to $\mathrm{Ca}^{2+}$ transporters leads to $\mathrm{Ca}^{2+}$ leakage into the cytosol and subsequent mitochondrial matrix $\mathrm{Ca}^{2+}$ overloading, which then leads to activation of the MPTP $[49,50]$.

Activation of the MPTP can also be seen as a product of increased ROS production. Notably, ROS production increases with age [51], and it is thought that ROS production is increased in complexes I and III with the inhibition of electron transport [52]. Oxidative damage to mtDNA and/or electron transport complexes is suggested to result in defective ROS-producing complexes. A cycle is established in which ROS produced by damaged mtDNA and/or electron transport complexes further damages electron transport complexes with age [51] (Figure 2). The increase in ROS production with age is noteworthy because increased MPTP activation is associated with elevated levels of ROS. This is based on the study conducted by Zorov and colleagues, who found that ROS accumulation within the mitochondria of cardiac myocytes leads to increased mitochondrial permeability transition and release of ROS from the mitochondria (ROSinduced ROS release) [53]. Thus, a clear relationship between age, elevated ROS levels, and increased mPTP openings is established. As will be discussed later, ROS released from the mitochondria can damage nuclear DNA and lead to proapoptotic signals which increase mPTP openings [5456]. Due to the scope of this article, changes in the respiratory chain with aging will not be discussed further.

ROS-induced ROS release is observed during aging and after injury. Inflammation, a process typically associated with injury, induces extracellular acidification [57]. This acidification can in turn lead to increased ROS production within the cell [58]. Increased ROS production in the cell instigates mROS release from the matrix of the mitochondria [9], specifically by means of the MPTP [59]. Thus, inflammation can effectively alter the function of the pore by increasing PT. These effects are not, however, limited to inflammation. Ischemia is also known to decrease extracellular $\mathrm{pH}$ [60], in turn launching the same ROS stimulating pathway described above in which the release of mROS further stimulates the production of ROS leading to a positive feedback mechanism in which normal pore function is disrupted [8].

Intracellular $\mathrm{pH}$, like extracellular $\mathrm{pH}$, plays a role in the interaction between inflammation, ischemia, and mPTP activation. Kerr et al. [61] used 2-deoxy-d-[3H]glucose (2-DG) mitochondrial entrapment to show that recovery of Langendorff-perfused rat hearts from ischemia is accompanied by a reversal of the mitochondrial PT [61]. This connection hinges on pyruvate, which is suggested to inhibit the mPTP by decreasing intracellular $\mathrm{pH}$. The beneficial effects of mPTP inhibition included recovery of left ventricular pressure [61]. When considering the results of their study, it is clear that MPTP function is altered in ischemic injury, specifically by means of increased permeability. Yet, it is this same alteration of increased permeability that further stimulates injury, as evidenced by the beneficial effects observed upon mPTP inhibition. The protective effects of mPTP inhibition are further evidenced by $\mathrm{Na}(+)-\mathrm{H}(+)$ exchanger-1 (NHE-1) inhibition. NHE-1 inhibition in hearts subjected to 


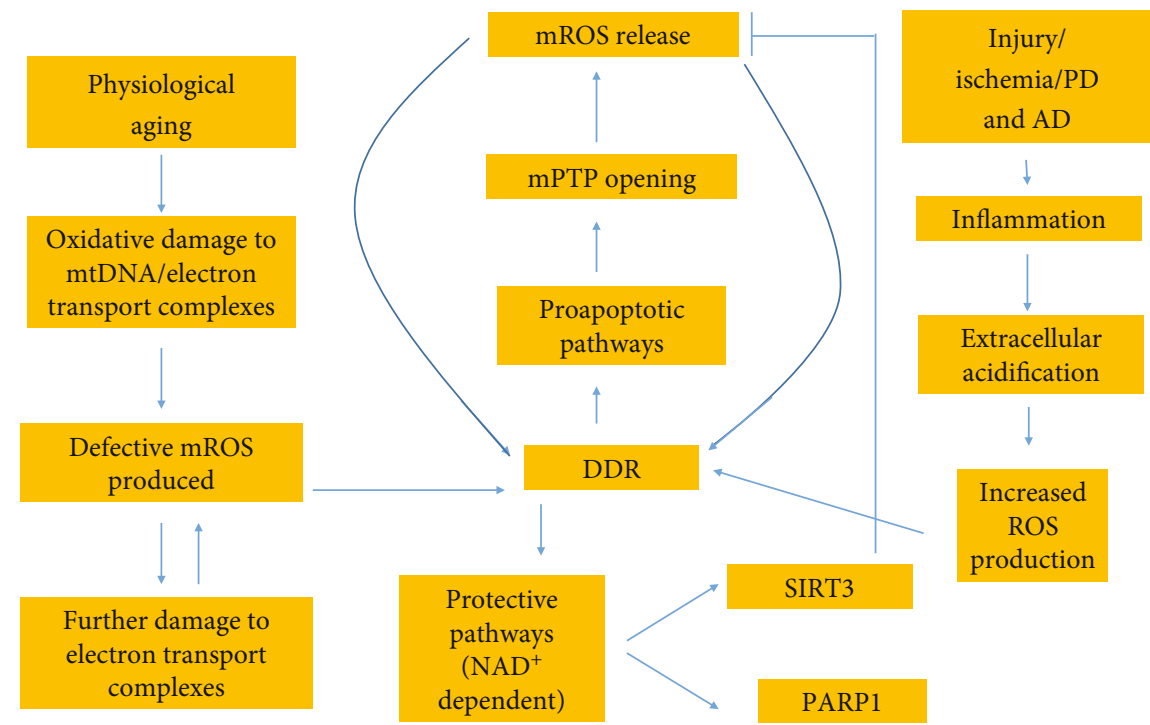

FIGURE 2: All of the following contribute to ROS production: physiological aging, injury, ischemia, $\mathrm{PD}$, and AD. Injury, ischemia, $\mathrm{PD}$, and $\mathrm{AD}$ do so by means of inducing inflammation. Extracellular acidification is a pathological effect of inflammation. A decrease in extracellular $\mathrm{pH}$ leads to increased ROS production within the cell which in turn instigates DDR. Aging results in oxidative damage to either mtDNA or electron transport complexes. This instigates defective mROS production. Upon ROS-induced ROS release, ROS can damage nuclear DNA, again inducing DDR. DDR results in proapoptotic pathways that induce mPTP opening and further mROS release. A positive feedback mechanism is initiated in which mPTP openings allow for mROS release which instigates DDR. Simultaneous to the proapoptotic mechanism is the $\mathrm{NAD}^{+}$-dependent protective pathways. SIRT3 in particular acts as an inhibitor to mROS release. It is important to note that these mechanisms are opposing and upon depletion of $\mathrm{NAD}^{+}$, the proapoptotic pathways dictate mROS release as the protective pathways are unable to perform their function.

ischemia/reperfusion using the same 2-DG mitochondrial entrapment method described above is associated with attenuation of mPTP opening [62]. The beneficial effects of mPTP attenuation also included recovery of left ventricular pressure [62]. A careful analysis of these studies shows that increased PT is observed with injury, inhibition of the MPTP can lead to a decrease in PT, and decreased PT can improve cardiac function.

ROS production within the cell leads to $\mathrm{MPTP}$ opening and subsequent mROS release. It is thought that the outermembrane anion channel, VDAC, plays a role in allowing the release of ROS from the intramembranous space of the mitochondria [63]. The ROS that are released by VDAC include superoxide and $\mathrm{H}_{2} \mathrm{O}_{2}$, as they are both small enough (less than $1500 \mathrm{kDa}$ ) to pass through the channel [64]. Once released into the cytosol, ROS damages nuclear DNA [11] and triggers the DNA damage response (DDR). DDR induces both proapoptotic signaling in postmitotic pathways [12] and protective pathways [11] (Figure 2). Proapoptotic signals include $\mathrm{p} 53$, which targets the mitochondrial matrix, and p66Shc, which targets the intermembrane space. p66Shc induces apoptosis specifically by means of generating $\mathrm{H}_{2} \mathrm{O}_{2} \cdot \mathrm{H}_{2} \mathrm{O}_{2}$ reacts with cytochrome $\mathrm{c}$ and induces oxidation of the MPTP leading to mitochondrial swelling and ultimately mPTP activation [54-56]. Thus, the increase in ROS production seen as a byproduct of aging initiates $\mathrm{mPTP}$ opening, but mPTP opening leads to further ROS production $\left(\mathrm{H}_{2} \mathrm{O}_{2}\right)$ via proapoptotic signals. This positive feedback mechanism is a means by which continued opening of the mPTP leads to a destruction of the membrane potential, swelling, and rupture of the outer mitochondrial membrane. The mPTP exacerbates the effects of aging as the rupture of the outer mitochondrial membrane leads to the release of ROS, $\mathrm{Ca}^{2+}$, and other metabolites which can, in turn, induce oxidative damage to proteins, transporters, and nuclear DNA ultimately disrupting cellular homeostasis $[9,50]$.

The frequency of mPTP opening is further increased by $\mathrm{Ca}^{2+}$ overloading in the matrix $[49,50,65] \mathrm{Ca}^{2+}$ concentration within the mitochondria is driven by cytosolic $\mathrm{Ca}^{2+}$ levels and mediated by the $\mathrm{Ca}^{2+}$ uniporter MCU $[66,67]$. It is known that aging disrupts $\mathrm{Ca}^{2+}$ homeostasis $[68,69]$ and interferes with the interaction between ER and mitochondria [70]. The disruption in $\mathrm{Ca}^{2+}$ homeostasis is thought to be a byproduct of oxidative damage to $\mathrm{Ca}^{2+}$ transporters which increases the leak of $\mathrm{Ca}^{2+}$ into the cytosol and subsequently increases $\mathrm{Ca}^{2+}$ overload of the mitochondria $[71,72]$ (Figure 3). Since oxidative damage to $\mathrm{Ca}^{2+}$ transporters is a byproduct of increased ROS levels, the continued opening of the MPTP would lead to further damage first initiated by cellular aging. In addition to damaged $\mathrm{Ca}^{2+}$ transporters, the direct transfer of calcium from the ER to the mitochondria increases $\mathrm{Ca}^{2+}$ overloading within the matrix [73]. To counter calcium overloading resulting from $\mathrm{mPTP}$ openings, MICU1, a subunit of MCU, limits calcium accumulation in the matrix as it maintains a threshold for calcium uptake $[66,74]$. In aged cells, however, cytosolic free calcium often exceeds the MICU1 threshold for calcium uptake while the calcium threshold controlling mPTP activation is lower than the normal threshold [75]. This would indicate that more $\mathrm{Ca}^{2+}$-induced mPTP openings are to be observed in aged 


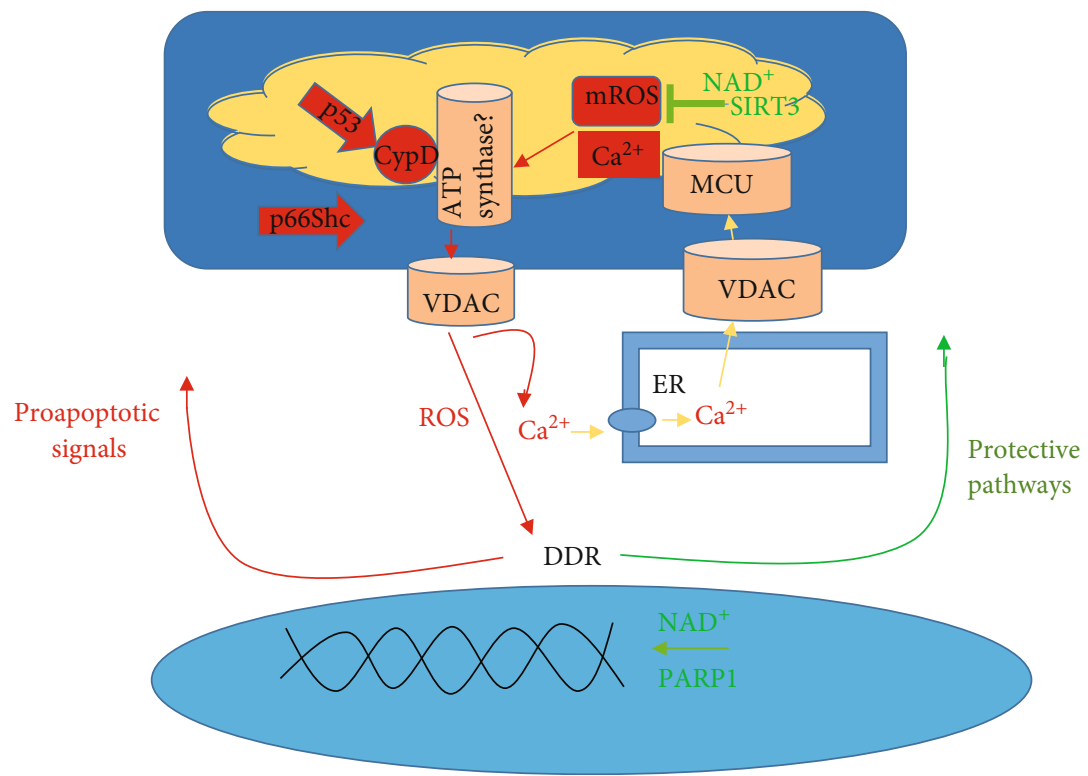

FIGURE 3: The components of the mPTP are of great controversy. However, despite this, CyPD and the controversial $\mathrm{F}_{1} \mathrm{~F}_{0}(\mathrm{~F})-\mathrm{ATP}$ synthase are shown as pore constituents. VDAC, while not considered to be part of the $\mathrm{mPTP}$, is thought to be how mROS, Ca ${ }^{2+}$, etc. are shuttled from the intermembrane space to the cytosol. mROS release through the mPTP leads to DNA and $\mathrm{Ca}^{2+}$ transporter damage. DNA damage induces DDR or DNA damage response. DDR subsequently induces both proapoptotic signals and protective pathways. Proapoptotic signals recruit p53 and p66Shc which act upon the MPTP (p53 specifically interacts with CypD, and p66Shc targets the intermembrane space generating ROS) to further induce mPTP openings. Oxidative damage to $\mathrm{Ca}^{2+}$ transporters can lead to calcium overloading and subsequent increased mPTP openings. MCU in particular can be affected by oxidative damage, leading to a disruption in mitochondrial Ca ${ }^{2+}$ levels. Protective pathways such as PARP1 aid in DNA repair, and SIRT3 inhibit mROS production. As further oxidative damage to DNA takes place, both protective pathways continue to utilize $\mathrm{NAD}^{+}$. $\mathrm{NAD}^{+}$depletion can result, leading to an inactivation of protective pathways. In turn, the proapoptotic signals are left unchallenged and $\mathrm{MPTP}$ openings become more frequent.

cells. Since ROS release can lead to oxidative damage of $\mathrm{Ca}^{2+}$ transporters and consequently $\mathrm{Ca}^{2+}$ overloading, increased mPTP sensitivity with age can be seen as a byproduct of both $\mathrm{Ca}^{2+}$ overloading and ROS release. Furthermore, mPTP opening can be seen as a key driver of the processes (oxidative damage to $\mathrm{Ca}^{2+}$ transporters, etc.) first initiated by aging.

\subsection{Protective Pathways Involving PARP1 and SIRT3 Can} Inhibit mPTP Opening. Although aging can increase ROS production, ROS do not always invoke damaging effects. This is because protective pathways exist to counter oxidative damage by ROS. Thus, due to the dual nature of ROS, which can have both protective and damaging effects, it is necessary to address the interplay between both to examine the overall effects of MPTP opening. Before the interplay can be discussed in regard to aging, it is necessary to examine the protective pathways stimulated by mROS that exist to maintain cellular homeostasis. One of the most important pathways begins with the intranuclear protein PARP1. When DNA is damaged through oxidative stress, PARP1 repairs DNA in a manner that is dependent on $\mathrm{NAD}^{+}[76]$. Working in conjunction with PARP1 are the sirtuins, which are similarly classified as NAD-dependent deacetylases [77]. It is thought that SIRT3 plays a particularly important role in inhibiting ROS production and mPTP activation in stem cells [78]. Through metabolic reprogramming, SIRT3 is suggested to effectively increase efficient electron transport away from carbohydrate catabolism, resulting in reduced ROS produc- tion $[79,80]$. Thus, because ROS production is decreased and MPTP activation is inhibited, the protective pathways above antagonize the MPTP from exacerbating the processes initiated by aging. With this in mind, it is clear that any downregulation of the protective pathways described above through depletion of $\mathrm{NAD}^{+}$would lead to the domination of MPTP openings and subsequent positive feedback regarding the release and production of mROS.

As aging proceeds, NAD+ depletion, mPTP openings, and mROS production and release lead to DNA damage. The protective pathways noted above, as well as activation of nuclear factor erythroid 2-related factor 2 (Nrf2), are initiated to counter oxidative damage; however, the continued activation of these pathways leads to a depletion of $\mathrm{NAD}^{+}$. Since both the PARP1 and sirtuin protective pathways are dependent upon $\mathrm{NAD}^{+}$, they are no longer able to perform their function as an inhibitor of mPTP opening and subsequent mROS release and production [81, 82]. Ultimately, as aging progresses, oxidative damage to nuclear DNA results in the activation of protective pathways which in turn leads to depletion of $\mathrm{NAD}^{+}$. Without $\mathrm{NAD}^{+}$, the protective pathways involving PARP1 and SIRT3 are unable to perform their function as an mPTP opening inhibitor. As a consequence, the MPTP can effectively exacerbate processes initiated by aging.

SIRT3 serves a protective role to inhibit ROS production but it can also act to deacetylate CypD and inhibit mPTP opening [83]. Since SIRT3 is dependent upon $\mathrm{NAD}^{+}$levels, 
CypD openings are therefore also dependent at least in part on $\mathrm{NAD}^{+}$levels. Consequently, as $\mathrm{NAD}^{+}$concentration declines as a byproduct of protection pathway activation, SIRT3 is unable to perform inhibition of CypD-induced mPTP opening [81, 83]. SIRT3's simultaneous effects on ROS production and CypD lead to an interplay between the two, which enables further mPTP openings. As discussed earlier, a decrease in SIRT3 activity leads to proapoptotic pathway activation through the ROS-induced DDR [12, 77]. In particular, $\mathrm{p} 53$ binds to CypD to form a complex triggering $\mathrm{mPTP}$ openings [44]. Thus, ROS production and CypD activation are connected via SIRT3 inhibition.

Pore openings are not limited to CypD's interaction with p53. Recently, a relationship was established between metformin, AMP kinase (AMPK), the peroxisome proliferatoractivated receptor- $\alpha(\operatorname{PPAR} \alpha) /$ mitochondria pathway, and CypD in cardiomyocytes [84]. It is known that activation of AMPK protects the heart from myocardial infarction and heart failure [84]. Thus, because it was found that metformin activates AMPK, metformin can be a potential impetus in driving myocardial protection. Metformin abolished oxidative stress-induced physical interactions between PPAR $\alpha$ and cyclophilin D (CypD), and the abolishment of these interactions was associated with inhibition of MPTP formation [84]. Thus, the myocardial protective effects of metformin were found to converge at the $\mathrm{mPTP}$.

\subsection{Aging, Decreased Lifespan, and Neurodegenerative} Diseases as Byproducts of mPTP Opening. MPTP openings become more frequent and longer in duration as a byproduct of increased ROS production with age and subsequent ROSinduced ROS release $[51,53]$. mPTP openings lead to the release of ROS which in turn stimulates proapoptotic pathways leading to further openings [12,54-56]. Due to the protective pathway dependence on $\mathrm{NAD}^{+}$, depletion of $\mathrm{NAD}^{+}$ leads to the inhibition of protective pathways leaving the counter effects of proapoptotic signals to proceed unchallenged $[81,83]$. Thus, it is necessary to address the effects of MPTP opening in regard to the overall phenomenon of aging. It has long been held that ROS accumulation leads to oxidative stress and the subsequent observable phenomenon of aging [85]. Recently, ROS involvement in cellular senescence has received significant attention with regard to organismal aging. Cellular senescence is thought to be initiated by genomic damage which activates DDR and subsequent pathways leading to growth arrest [86]. The accumulation of senescent cells in organismal tissue is commensurate with advancing age, and senescent cells reduce stem and progenitor cell numbers leading to impaired capacity for tissue regeneration [87-89]. Considering that ROS play a vital role in cellular senescence and the MPTP plays a vital role in the release of ROS, it is therefore likely that the MPTP contributes to the progression of senescence.

Despite knowledge on the interaction between ROS and the MPTP, little work has been done with regard to the relation between the mPTP and cellular senescence. Hofer and colleagues investigated rat ventricular subsarcolemmal (SSM) and interfibrillar (IFM) mitochondrial susceptibility to $\mathrm{Ca}^{2+}$-induced $\mathrm{mPTP}$ openings with aging and calorie restriction [90]. They found that IFM exhibited an increased susceptibility towards mPTP openings during senescence. A decline in $\mathrm{Ca}^{2+}$ retention was observed with aging, particularly during senescence [90]. It is important to note that SSM did not exhibit these same results, and mPTP's association with senescence may be dependent on the tissue type. SSM aside, these results would suggest that the MPTP plays a role in the induction of cellular senescence and thus tissue aging, as evidenced by the decline in $\mathrm{Ca}^{2+}$ retention. As described above, genomic damage initiates the cellular pathway inducing senescence. Since it is known that ROS release through the MPTP is capable of inducing DDR, it is clear that a relation between $\mathrm{mPTP}$ opening, induction of cellular senescence, and cell and tissue aging exists.

Another proposed mechanism by which mPTP opening leads to cellular aging is through increased levels of autophagy. While autophagy is commonly thought to increase longevity due to its ability to clear damaged proteins and dysfunctional organelles, it can be detrimental at very high levels [91]. Elevated autophagy shortened lifespan in C. elegans lacking serum/glucocorticoid-regulated kinase-1 (sgk1) because of increased mitochondrial permeability [91]. Furthermore, mice maintaining sgk-1 displayed lower levels of mitochondrial permeability, normal levels of autophagy, and normal lifespan. Based on these results, sgk-1 is suggested to modulate mPTP opening, which in turn mediates mitochondrial permeability, autophagy, and lifespan [91]. Since mitochondrial permeability is enhanced in the absence of sgk-1, it can be concluded that lifespan reduction as a byproduct of elevated autophagy is likely due to increased mPTP activity.

Research on neurodegenerative diseases, particularly on $\mathrm{PD}$, has uncovered significant findings regarding $\mathrm{mPTP}$ openings and aging. PD is characterized by two phenomena including loss of dopaminergic neurons in the substantia nigra [92] and accumulation of highly insoluble fibrillar aggregates of the protein alpha-synuclein [93]. Recently, Ludtmann and colleagues [64] investigated the relationship between monomeric and oligomeric $\alpha$-synuclein encoded by the gene SNCA and their subsequent effects on $\mathrm{mPTP}$ openings and cellular death. While $\alpha$-synuclein in its monomeric form improves ATP synthase efficiency, upon protein aggregation and subsequent formation of the oligomeric form, a toxic gain of function is observed. Specifically, as it relates to the $\mathrm{MPTP}$, the oligomers induce selective oxidation of the ATP synthase beta subunit resulting in an increased probability of $\mathrm{MPTP}$ opening. This finding is significant as induced pluripotent stem cell- (iPSC-) derived neurons bearing SNCA triplication generate $\alpha$-synuclein aggregates that interact with ATP synthase and induce mPTP opening, leading to neuronal death [94].

PD is, however, not fully characterized by neuronal death alone. Loss of the antioxidant protein (protein-disulfide reductase) glutathione (GSH), a reduction in mitochondrial complex I activity, increased oxidative damage of DNA, and elevated free iron levels in the substania nigra have all been documented in patients suffering from PD $[95,96]$. As mentioned earlier, ROS production increases with age, specifically in complexes I and III with the inhibition of electron 
transport [51, 52]. Furthermore, ROS accumulation within the mitochondria can lead to ROS-induced ROS release via the MPTP [53]. ROS released from the mitochondria can damage nuclear DNA and lead to proapoptotic signals which stimulate further mPTP openings [54-56]. Thus, the mPTP links two key processes associated with PD: a reduction in mitochondrial complex I activity leading to increased mitochondrial ROS, which in turn prompts mPTP openings and subsequent ROS release inducing increased DNA damage [51-56]. It is also important to note that neuroinflammation is observed in PD [64], and inflammation leads to extracellular acidification [33] which in turn leads to increased ROS production in the cell driving further mROS release from the matrix of the mitochondria via the $\operatorname{mPTP}[5,34,35]$. The etiology of PD is complex and multifactorial involving environmental factors, genetic susceptibility, and aging that together promote disease progression [97]. The findings reviewed above suggest that the $\mathrm{MPTP}$ is also likely to have a potential role in the pathogenesis of PD.

Age-related dysfunctions of the MPTP extend to and are prevalent in age-related pathologies mediated by various factors such as inflammation. Inflammation is an early step in the pathogenesis of $\mathrm{AD}$ [98], and neuroinflammation is a process that occurs in PD [99]. As was noted earlier, extracellular acidification can increase ROS production, which leads to increased PT via the opening of the mPTP [59]. MPTP dysfunction may also be involved in the progression of $\mathrm{AD}$. In its later stages, $\mathrm{AD}$ is characterized by massive amyloidbeta $(\mathrm{A} \beta)$ deposition in the parenchyma and the cerebrovascular walls $[100,101]$. Recent findings show that mitochondrial damage in $\mathrm{AD}$ is linked to $\mathrm{A} \beta$ toxicity [102-105]. Some examples include decreased mitochondrial respiratory chain function $[105,106]$, increased mitochondrial ROS generation [105, 107], and changes in mitochondrial structure [108]. The interaction of $\mathrm{A} \beta$ species with certain regulators of the MPTP is likely responsible for the aforementioned damage. Specifically, the interaction of $\mathrm{A} \beta$ species with CypD and the upregulation of $\mathrm{CypD}$ expression were found to decrease the threshold of mPTP activation [109]. An AD mouse model overexpressing a mutant human form of amyloid precursor protein (mAPP) has also been shown to demonstrate increased CypD levels [109]. Thus, $\mathrm{A} \beta$ appears to be an important mediator connecting $\mathrm{AD}$ to the $\mathrm{mPTP}$.

CypD is considered a crucial component for mitochondrial permeability transition pore (mPTP) formation [4, 110]. Du et al. found that mitochondrial function and learning/memory were significantly improved in CypDdeficient mice $[109,111]$. These results suggest that pore formation is a necessary step in the pathogenesis of $\mathrm{AD}$ and that the ablation of CypD in mice gives lifelong protection against $\mathrm{A} \beta$-induced mitochondrial and behavioral dysfunction [111]. Other studies have shown that $\mathrm{A} \beta$ oligomers induce a massive entry of $\mathrm{Ca}(2+)$ in neurons and promote mitochondrial $\mathrm{Ca}(2+)$ overload and mitochondrial PT [112]. This is significant because, as mentioned earlier, $\mathrm{Ca}^{2+}$ overload can lead to mPTP activation [49, 50]. Nonsteroidal antiinflammatory drugs (NSAIDs), including salicylate and sulindac sulfide, were able to inhibit mitochondrial $\mathrm{Ca}^{2+}$ overload through mitochondrial depolarization. These stud- ies highlight the role of MPTP dysfunction in neurodegenerative disease.

1.5. Potential Therapies to Mitigate mPTP Opening. Previous work suggests that $\mathrm{mPTP}$ opening plays a role in both injury and aging, thus targeted therapies to inhibit continued and frequent opening of the MPTP may serve to promote longevity and healthspan (Table 1). As discussed earlier, PD, AD, and other age-related disorders are thought to be byproducts of mPTP openings. Research targeting the mPTP whether directly or indirectly is divided into two areas. The first area involves therapeutics that require some form of interaction with CypD, and the second area involves therapeutics that require no interaction with $\mathrm{CypD}$ [113].

Of the therapies that inhibit CypD, cyclosporin A (CsA) has evoked great interest as it has shown cytoprotective properties in cellular models due to its ability to interfere with the interaction of CypD with the mPTP [114]. Specifically, CsA has been shown to block mitochondrial $\mathrm{Ca}^{2+}$ efflux and allow mitochondria to accumulate large amounts of $\mathrm{Ca}^{2+}$ [115]. The mechanism which is responsible for increased $\mathrm{Ca}^{2+}$ retention is indicative of $\mathrm{mPTP}$ closure [45]. This point is supported by Crompton and colleagues, who found that ability of mitochondria to retain $\mathrm{Ca}^{2+}$ in the presence of CsA was due to CsA inhibition of the mPTP [116]. CypD in particular was shown to be the target of CsA [117]. mPTP openings were studied in ischemic reperfusion injury in rat hearts to determine the efficacy of CsA with regard to cardioprotection. Cardioprotection was observed in a narrow range, between 0.2 and $0.4 \mu \mathrm{M}$, as benefits were lost at concentrations above $0.4 \mu \mathrm{M}$ [118]. Despite these promising results, a recent clinical trial showed that CsA failed to improve clinical outcomes and prevent adverse left ventricular remodeling in patients with an acute anterior ST-segment elevation myocardial infarction (STEMI) [117]. This raises questions regarding the viability of targeting CypD to promote cardioprotection. It is possible that these seemingly conflicting studies on the cardioprotection offered by CsA could be explained by the means of drug administration. Since cardioprotection was observed in a narrow range in rat hearts, it is possible that the dosage administered in the clinical trial, $2.5 \mathrm{mg} / \mathrm{kg}$ body weight, was too low/high of a concentration [117].

Further research supporting CypD as a viable cardioprotective target was conducted by Parodi-Rullman et al. on induced myocardial infarction in rats [119]. They found that CypD inhibition exerts cardioprotective effects in reperfused but not in nonreperfused infarcted hearts of female rats, and the effects are observed only during acute postinfarction injury. CypD remains a viable target for age-related pathologies, although the timing and dosage of drug administration should be refined and optimized to demonstrate clear benefits for the patient. The CypD inhibitor, CsA derivative $\mathrm{N}$ methyl-isoleucine-4-cyclosporin (NIM811), has been investigated as a therapeutic alternative to CsA alone. In a study conducted to determine the efficacy of NIM811 with regard to inhibiting the MPTP, it was found that both mitochondrial permeability transition onset and apoptosis were prevented when NIM811 was added to rat hepatocytes [114]. The 
TABLE 1: Small molecules targeting the mPTP, directly or indirectly, and their potential impact on age-associated diseases.

\begin{tabular}{|c|c|c|c|}
\hline Classification & Compound & Experimental model & Effect \\
\hline $\begin{array}{l}\text { CypD inhibition } \\
\text { independent }\end{array}$ & Melatonin & Rat stroke model & $\begin{array}{l}\text { Decreased neuron loss and reduced } \\
\text { infarct volume }[124]\end{array}$ \\
\hline \multirow{2}{*}{$\begin{array}{l}\text { CypD inhibition } \\
\text { dependent }\end{array}$} & Cyclosporin A (CsA) & $\begin{array}{l}\text { Ischemic reperfusion injury rat heart } \\
\text { [118]; anoxia-induced injury in rat heart } \\
\text { myocytes [139] }\end{array}$ & $\begin{array}{l}\text { Cardioprotection [118]; reduced } \\
\text { proportion of necrosis in rat heart } \\
\text { myocytes [139] }\end{array}$ \\
\hline & & Isolated mitochondria in rat hepatocytes & \\
\hline
\end{tabular}

N-Methyl-isoleucine-4cyclo-sporin (NIM811)

(E)-3-(4-Fluoro-3-hydroxyphenyl)-N-naphthalen-1-yl-

CypD inhibition independent acrylamide (compound 22)

\section{Edaravone (Radicut ${ }^{\mathrm{TM}}$ )}

Ischemia/reperfusion injury in rat brain

N-Phenylbenzamides (CypD inhibition independent)

Isoxazoles (CypD inhibition independent)

Cinnamic anilides (CypD inhibition independent)

SS-31

XJB-5-131

Electron

scavengers/antioxidants (CypD inhibition independent)

MitoQ

EUK-8

(TNF $\alpha$-induced permeable transition onset)

Rabbit model of acute myocardial infarction

Compound 4, (3-

(benzyloxy)-5-chloro-N-(4- $\mathrm{Ca}^{2+}$ retention capacity (CRC) assay in (piperidin-1-

ylmethyl)phenyl)benzamide)

Compound 1, 5-(3-

hydroxyphenyl)-N-(3,4,5-

trimethoxyphenyl)isoxazole3-carboxamide

GNX-4728

Mouse model of amyotrophic lateral sclerosis (ALS)

15-month-old male mice exposed to isoflurane [141]; antioxidant properties of SS peptides in neuronal $\mathrm{N}_{2} \mathrm{~A}$ cells treated with t-butylhydroperoxide (tBHP) [131]

MPTP openings measured by CRC in isolated mouse liver mitochondria

Cardiolipin oxidation as a byproduct of experimental traumatic brain injury in rats [133]; cardiac function in aged rats [132]; muscle contractility of aged adult rats [142]

Cardiac ischemia-reperfusion injury in rats

Oxidative stress-sensitized harlequin (Hq) mutant mice and their wild-type (WT) counterparts [143]; presymptomatic heart/muscle-specific manganese-superoxide dismutase(Mn-SOD-) deficient mice [135]
Mitochondrial permeability transition onset and apoptosis prevented [114]

Cardioprotective; reduced infarct size; inhibitor of mPTP openings [127]

Neuroprotective; inhibited $\mathrm{Ca}^{2+}$ - and $\mathrm{H}_{2} \mathrm{O}_{2}$-induced swelling in mitochondria; inhibited $\mathrm{Ca}^{2+}$ generation of ROS [140]

Induced a concentration-dependent increase in the CRC of permeabilized

HeLa cells (indicative of MPTP inhibition) [129]

Inhibitory activity against mitochondrial swelling; no interference on the inner mitochondrial membrane potential [130]

Slowed disease progression and significantly improved motor function; displayed a nearly 2 -fold extension of lifespan; established mitochondrial calcium retention [128]

Enhances synaptic plasticity and provides protective effects on cognitive function [141]; reduced intracellular ROS and increased cell survival [131]

Inhibition of cardiolipin oxidation and subsequent improvement in motor skills and cognitive operations [133];

improved postischemic recovery of aged hearts, reduced $\mathrm{Ca}(2+)$-induced swelling in the mitochondria, attenuated the $\mathrm{H}_{2} \mathrm{O}_{2}$-induced depolarization of the mitochondrial inner membrane as well as the total and mitochondrial ROS levels in cultured cardiomyocytes [132]; higher muscle contractility [142]

Decreased heart dysfunction, cell death, and mitochondrial damage after ischemia-reperfusion [134]

Improved left ventricular end-systolic dimensions and fractional shortening, prevented myocardial oxidant stress, attenuated necrotic and apoptotic cell death, and attenuated cardiac hypertrophy and fibrosis in both $\mathrm{Hq}$ and WT [143]; suppressed the progression of cardiac dysfunction and diminished ROS production and oxidative damage [135] 
TABLe 1: Continued.

\begin{tabular}{|c|c|c|c|}
\hline Classification & Compound & Experimental model & Effect \\
\hline & MitoTEMPO & $\begin{array}{l}\text { Amyloid-beta toxicity in primary } \\
\text { cultured mouse neurons }\end{array}$ & $\begin{array}{l}\text { Neuronal lipid oxidation was } \\
\text { significantly suppressed; demonstrated } \\
\text { protective effects on mitochondrial } \\
\text { bioenergetics evidenced by preserved } \\
\text { mitochondrial membrane potential and } \\
\text { cytochrome c oxidase activity as well as } \\
\text { ATP production [136] }\end{array}$ \\
\hline
\end{tabular}

potential of NIM811 for reducing mitochondrial permeability and improving cell survival has also been shown in animal models of spinal cord injury [120], traumatic brain injury [121], and hindlimb ischemia-reperfusion injury [122].

CypD-independent therapeutics have received attention (Table 1). Melatonin in particular has been studied as a potential inhibitor to the MPTP that does not require CypD interaction. Melatonin has been shown to inhibit MPTP activation as evidenced by reduced mitochondrial swelling and increased $\mathrm{Ca}^{2+}$ capacity [123]. This is further supported by Andrabi et. al who studied the effects of melatonin on mPTP openings in rat brain models [124]. The release of cytochrome $\mathrm{c}$ was used to assess pore opening, and rats treated with melatonin displayed a marked decrease in cytochrome $c$ release [124]. These results would support the assertion that melatonin does indeed inhibit mPTP activation. Postmortem analyses of cerebrospinal fluid shows a marked decrease in melatonin concentration with age [125], which could in theory contribute to increased $\mathrm{MPTP}$ opening with aging. $\mathrm{MPTP}$ openings lead to swelling of the mitochondria, rupture of the outer mitochondrial membrane, and subsequent release of intermembranous proteins [126]. Melatonin supplementation may therefore represent one option to suppress $\mathrm{mPTP}$ opening in older adults who are likely to have relatively low endogenous levels of melatonin.

In addition to melatonin, other CypD-independent therapeutics include mitotargeted compounds (Table 1). Mitotargeted therapeutics acting in the absence of CypD interaction include electron scavengers, cinnamic anilides, $\mathrm{N}$-phenylbenzamides, and isoxazoles. One small molecule that directly targets the mPTP is (E)-3-(4-fluoro-3hydroxy-phenyl)-N-naphthalen-1-yl-acrylamide (compound 22), a cinnamic anilide that inhibits oxidative stress and chemical crosslinker-induced mPTP opening [127]. Other novel CypD-independent therapies exist, classified in the same cinnamic anilide series and performing similar functions as compound 22. One example is GNX-4728 which was administered in a mouse model of amyotrophic lateral sclerosis (ALS). GNX-4728 was found to slow disease progression, improve motor function, and extend lifespan by nearly twofold. Furthermore, $\mathrm{Ca}^{2+}$ retention was established, which is again indicative of $\mathrm{mPTP}$ closure [128]. Regarding $\mathrm{N}$-phenylbenzamides, the most prominent therapeutic candidate is compound 4, (3-(benzyloxy)-5-chloro- $\mathrm{N}$-(4-(piperidin-1-ylmethyl)phenyl)benzamide). Compound 4 induced a concentration-dependent increase in the calcium retention capacity (CRC) of permeabilized HeLa cells suggesting mPTP inhibition [129]. The isoxazole, compound 1, 5-(3-
hydroxyphenyl)-N-(3,4,5-trimethoxyphenyl)isoxazole-3carboxamide, produced similar results in an isolated mouse liver mitochondria model. Compound 1 was shown to inhibit mitochondrial swelling without interfering with the inner mitochondrial membrane potential [130].

Electron scavengers are mitotargeted therapeutics acting in the absence of CypD interaction. Some of the most studied drug therapies in this category include SS-31, XJB-5-131, MitoQ, EUK-8, and MitoTEMPO. SS-31 can increase cell survival and reduce intracellular ROS in neuronal N2A cells treated with t-butylhydroperoxide (tBHP) [131]. XJB-5-131 can improve postischemic recovery of aged hearts, reduce $\mathrm{Ca}^{2+}$-induced swelling in mitochondria, and reduce total mROS levels in cardiomyocytes [132]. It was also observed that XJB-5-131 improved motor skills and cognitive functions in rats with traumatic brain injury. These results are seen as a byproduct of decreased levels of mROS and subsequent prevention of cardiolipin oxidation [133]. Both MitoQ and EUK-8 employ the same electron-scavenging mechanisms as the therapies above. MitoQ's therapeutic effects were examined in rat cardiac ischemia-reperfusion injury and MitoQ decreased cell death and decreased mitochondrial damage [134]. EUK-8's effects were examined in presymptomatic heart/muscle-specific manganese-superoxide dismutase- (Mn-SOD-) deficient mice. It was observed that EUK-8 suppressed the progression of cardiac dysfunction and diminished ROS production and oxidative damage [135]. Again, while the above therapies do not interact directly with the mPTP, they do reduce ROS levels or production within the mitochondria, which leads to inhibition of mPTP opening.

MitoTEMPO has been investigated as a potential therapeutic in the treatment of $\mathrm{AD}$. A recent study was performed in which $\mathrm{A} \beta$ toxicity, a hallmark of $\mathrm{AD}$, was measured in primary cultured mouse neurons. Upon treatment with MitoTEMPO, it was found that $\mathrm{A} \beta$-induced mitochondrial superoxide production and neuronal lipid oxidation were significantly decreased. Furthermore, a protective effect on mitochondrial bioenergetics was observed as evidenced by preserved mitochondrial membrane potential [136]. These results would indicate that MitoTEMPO has the potential to protect neuronal function in $\mathrm{AD}$. While the previous therapies are in the developing stages, one therapy had been approved for treating acute ischemic stroke in Japan. Edaravone is a free radical scavenger that produces neuroprotective effects. The mechanism in which this is accomplished is similar to the other scavengers in that edaravone captures and reduces excessive ROS [137]. Similarly, as with the other 
scavengers, the therapy acts on the relationship between ROS and MPTP activation. Thus, as a byproduct of edaravone administration, a decrease in ROS is observed and a decrease in mPTP activation occurs.

\section{Summary and Conclusions}

Mitochondrial dysfunction is now thought to play a significant role in the tissue degeneration and loss of function that occurs in multiple organ systems with advanced age. A key factor in this process is the generation of reactive oxygen species in mitochondria of aged cells, which is in turn associated with cell death, senescence, and tissue damage. The mitochondrial permeability transition pore appears to play a significant role in ROS production with aging. For example, the continued opening of MPTP and release of mROS lead to DNA damage. Cytoprotective pathways are activated to counter oxidative damage; however, the continued activation of these pathways leads to a depletion of NAD+. Since both the PARP1 and sirtuin protective pathways are dependent on NAD+, they lose their ability to suppress MPTP opening and inhibit mROS release and production. These findings point to $\mathrm{mPTP}$ inhibition as a potential therapeutic target for age-related disorders. Mitotargeted compounds and small molecules such as NIM811 have, at least in animal models, demonstrated success in promoting cell survival in settings associated with significant cellular damage such as spinal cord injury, traumatic brain injury, and ischemic stroke. Despite this, the application of MPTP targeted drugs in a medical setting remains elusive. This is evidenced by CsA, which failed to improve clinical outcomes and prevent adverse left ventricular remodeling in patients with an acute myocardial infarction. The electron scavenger edaravone remains one of the only mPTP-targeted drugs approved for clinical use as a neuroprotective agent. Future studies should be directed at exploring more long-term use of these small molecules in older animals to determine their effects on the development and progression of chronic age-associated disorders of the brain, musculoskeletal, and cardiovascular systems.

\section{Conflicts of Interest}

The authors declare that they have no conflicts of interest.

\section{Acknowledgments}

Funding for this research was provided by the National Institute on Aging, US National Institutes of Health (AG036675), and US Army Medical Research and Materiel Command CDMRP Program Grant DM160252.

\section{References}

[1] N. Sun, R. J. Youle, and T. Finkel, "The mitochondrial basis of aging," Molecular Cell, vol. 61, no. 5, pp. 654-666, 2016.

[2] D. V. Ziegler, C. D. Wiley, and M. C. Velarde, "Mitochondrial effectors of cellular senescence: beyond the free radical theory of aging," Aging Cell, vol. 14, no. 1, pp. 1-7, 2015.
[3] S. I. Liochev, "Reactive oxygen species and the free radical theory of aging," Free Radical Biology \& Medicine, vol. 60, pp. 1-4, 2013.

[4] C. P. Baines, R. A. Kaiser, N. H. Purcell et al., "Loss of cyclophilin D reveals a critical role for mitochondrial permeability transition in cell death," Nature, vol. 434, no. 7033, pp. 658662, 2005.

[5] R. A. Eliseev, G. Filippov, J. Velos et al., "Role of cyclophilin D in the resistance of brain mitochondria to the permeability transition," Neurobiology of Aging, vol. 28, no. 10, pp. 15321542, 2007.

[6] J. Jordán, V. Ceña, and J. H. Prehn, "Mitochondrial control of neuron death and its role in neurodegenerative disorders," Journal of Physiology and Biochemistry, vol. 59, no. 2, pp. 129-141, 2003.

[7] C. P. Connern and A. P. Halestrap, "Recruitment of mitochondrial cyclophilin to the mitochondrial inner membrane under conditions of oxidative stress that enhance the opening of a calcium-sensitive non-specific channel," The Biochemical Journal, vol. 302, no. 2, pp. 321-324, 1994.

[8] H. Rottenberg and J. B. Hoek, "The path from mitochondrial ROS to aging runs through the mitochondrial permeability transition pore," Aging Cell, vol. 16, no. 5, pp. 943-955, 2017.

[9] D. B. Zorov, M. Juhaszova, and S. J. Sollott, "Mitochondrial reactive oxygen species (ROS) and ROS-induced ROS release," Physiological Reviews, vol. 94, no. 3, pp. 909-950, 2014.

[10] C. E. Schaar, D. J. Dues, K. K. Spielbauer et al., "Mitochondrial and cytoplasmic ROS have opposing effects on lifespan," PLoS Genetics, vol. 11, no. 2, article e1004972, 2015.

[11] E. F. Fang, M. Scheibye-Knudsen, K. F. Chua, M. P. Mattson, D. L. Croteau, and V. A. Bohr, "Nuclear DNA damage signalling to mitochondria in ageing," Nature Reviews. Molecular Cell Biology, vol. 17, no. 5, pp. 308-321, 2016.

[12] S. Nicolai, A. Rossi, N. di Daniele, G. Melino, M. Annicchiarico-Petruzzelli, and G. Raschellà, "DNA repair and aging: the impact of the p53 family," Aging, vol. 7, no. 12, pp. 1050-1065, 2015.

[13] J. E. Chipuk, L. Bouchier-Hayes, and D. R. Green, "Mitochondrial outer membrane permeabilization during apoptosis: the innocent bystander scenario," Cell Death and Differentiation, vol. 13, no. 8, pp. 1396-1402, 2006.

[14] V. K. Rao, E. A. Carlson, and S. S. Yan, "Mitochondrial permeability transition pore is a potential drug target for neurodegeneration," Biochimica et Biophysica Acta, vol. 1842, no. 8, pp. 1267-1272, 2014.

[15] K. Kalani, S. F. Yan, and S. S. Yan, "Mitochondrial permeability transition pore: a potential drug target for neurodegeneration," Drug Discovery Today, vol. 23, no. 12, pp. 1983-1989, 2018.

[16] J. Doczi, B. Torocsik, A. Echaniz-Laguna et al., "Alterations in voltage-sensing of the mitochondrial permeability transition pore in ANT1-deficient cells," Scientific Reports, vol. 6, no. 1, article 26700, pp. 1-21, 2016.

[17] P. Bernardi, "Why F-ATP synthase remains a strong candidate as the mitochondrial permeability transition pore," Frontiers in Physiology, vol. 9, 2018.

[18] V. Giorgio, V. Burchell, M. Schiavone et al., " $\mathrm{Ca}^{2+}$ binding to F-ATP synthase $\beta$ subunit triggers the mitochondrial permeability transition," EMBO Reports, vol. 18, no. 7, pp. 10651076, 2017. 
[19] N. Mnatsakanyan, M. C. Llaguno, Y. Yang et al., "A mitochondrial megachannel resides in monomeric $\mathrm{F}_{1} \mathrm{~F}_{\mathrm{O}}$ ATP synthase," Nature Communications, vol. 10, no. 1, 2019.

[20] V. Giorgio, S. von Stockum, M. Antoniel et al., "Dimers of mitochondrial ATP synthase form the permeability transition pore," Proceedings of the National Academy of Sciences of the United States of America, vol. 110, no. 15, pp. 58875892, 2013.

[21] M. Bonora, A. Bononi, E. de Marchi et al., "Role of the c subunit of the FOATP synthase in mitochondrial permeability transition," Cell Cycle, vol. 12, no. 4, pp. 674-683, 2014.

[22] K. N. Alavian, G. Beutner, E. Lazrove et al., "An uncoupling channel within the c-subunit ring of the F1FO ATP synthase is the mitochondrial permeability transition pore," Proceedings of the National Academy of Sciences of the United States of America, vol. 111, no. 29, pp. 10580-10585, 2014.

[23] M. Carraro, V. Giorgio, J. Šileikyte et al., "Channel formation by yeast F-ATP synthase and the role of dimerization in the mitochondrial permeability transition," The Journal of Biological Chemistry, vol. 289, no. 23, pp. 1598015985, 2014.

[24] S. von Stockum, V. Giorgio, E. Trevisan et al., "F-ATPase of Drosophila melanogaster Forms 53-Picosiemen (53-pS) Channels Responsible for Mitochondrial $\mathrm{Ca}^{2+}$-induced $\mathrm{Ca}^{2+}$ Release*," The Journal of Biological Chemistry, vol. 290, no. 8, pp. 4537-4544, 2015.

[25] M. Antoniel, K. Jones, S. Antonucci et al., "The unique histidine in OSCP subunit of F-ATP synthase mediates inhibition of the permeability transition pore by acidic $\mathrm{pH}$," $E M B O$ Reports, vol. 19, no. 2, pp. 257-268, 2018.

[26] L. Guo, M. Carraro, G. Sartori et al., "Arginine 107 of yeast ATP synthase subunit g mediates sensitivity of the mitochondrial permeability transition to phenylglyoxal," The Journal of Biological Chemistry, vol. 293, no. 38, pp. 14632-14645, 2018.

[27] M. Carraro, V. Checchetto, G. Sartori et al., "High-conductance channel formation in yeast mitochondria is mediated by F-ATP synthase e and g subunits," Cellular Physiology and Biochemistry, vol. 50, no. 5, pp. 1840-1855, 2018.

[28] J. He, H. C. Ford, J. Carroll, S. Ding, I. M. Fearnley, and J. E. Walker, "Persistence of the mitochondrial permeability transition in the absence of subunit c of human ATP synthase," Proceedings of the National Academy of Sciences of the United States of America, vol. 114, no. 13, pp. 3409-3414, 2017.

[29] J. He, J. Carroll, S. Ding, I. M. Fearnley, and J. E. Walker, "Permeability transition in human mitochondria persists in the absence of peripheral stalk subunits of ATP synthase," Proceedings of the National Academy of Sciences of the United States of America, vol. 114, no. 34, pp. 90869091, 2017.

[30] S. Massari and G. F. Azzone, "The equivalent pore radius of intact and damaged mitochondria and the mechanism of active shrinkage," Biochimica et Biophysica Acta, vol. 283, no. 1, pp. 23-29, 1972.

[31] A. Scarpa and G. F. Azzone, "The mechanism of ion translocation in mitochondria. 4. Coupling of $\mathrm{K}^{+}$efflux with $\mathrm{Ca}^{2+}$ uptake," European Journal of Biochemistry, vol. 12, no. 2, pp. 328-335, 1970.

[32] D. E. Wingrove, J. M. Amatruda, and T. E. Gunter, “Glucagon effects on the membrane potential and calcium uptake rate of rat liver mitochondria," The Journal of Biological Chemistry, vol. 259, no. 15, pp. 9390-9394, 1984.
[33] M. Bragadin, T. Pozzan, and G. F. Azzone, "Kinetics of calcium $^{2+}$ ion carrier in rat liver mitochondria," Biochemistry, vol. 18, no. 26, pp. 5972-5978, 1979.

[34] D. G. Nicholls, "The regulation of extramitochondrial free calcium ion concentration by rat liver mitochondria," The Biochemical Journal, vol. 176, no. 2, pp. 463-474, 1978.

[35] A. Nicolli, V. Petronilli, and P. Bernardi, "Modulation of the mitochondrial cyclosporin A-sensitive permeability transition pore by matrix $\mathrm{pH}$. Evidence that the pore open-closed probability is regulated by reversible histidine protonation," Biochemistry, vol. 32, no. 16, pp. 4461-4465, 1993.

[36] N. Brustovetsky, M. Klingenberg, E. Bamberg, and A. Becker, "Electrical currents associated with nucleotide transport by the reconstituted mitochondrial ADP/ATP carrier," Proceedings of the National Academy of Sciences of the United States of America, vol. 93, no. 2, pp. 664-668, 1996.

[37] N. Brustovetsky, M. Tropschug, S. Heimpel, D. Heidkämper, and $\mathrm{M}$. Klingenberg, "A large $\mathrm{Ca}^{2+}$-dependent channel formed by recombinant ADP/ATP carrier from Neurospora crassa resembles the mitochondrial permeability transition pore," Biochemistry, vol. 41, no. 39, pp. 11804-11811, 2002.

[38] A. W. Leung, P. Varanyuwatana, and A. P. Halestrap, "The Mitochondrial Phosphate Carrier Interacts with Cyclophilin D and May Play a Key Role in the Permeability Transition," The Journal of Biological Chemistry, vol. 283, no. 39, pp. 26312-26323, 2008.

[39] P. Varanyuwatana and A. P. Halestrap, "The roles of phosphate and the phosphate carrier in the mitochondrial permeability transition pore," Mitochondrion, vol. 12, no. 1, pp. 120-125, 2012.

[40] S. Nesci, "The mitochondrial permeability transition pore in cell death: a promising drug binding bioarchitecture," Medicinal Research Reviews, vol. 40, no. 2, pp. 811-817, 2020.

[41] E. Gauba, L. Guo, and H. Du, "Cyclophilin D promotes brain mitochondrial F1FO ATP synthase dysfunction in aging mice," Journal of Alzheimer's Disease, vol. 55, no. 4, pp. 1351-1362, 2017.

[42] A. Folda, A. Citta, V. Scalcon et al., "Mitochondrial thioredoxin system as a modulator of cyclophilin D redox state," Scientific Reports, vol. 6, no. 1, article 23071, 2016.

[43] T. T. Nguyen, M. V. Stevens, M. Kohr, C. Steenbergen, M. N. Sack, and E. Murphy, "Cysteine 203 of Cyclophilin D Is Critical for Cyclophilin D Activation of the Mitochondrial Permeability Transition Pore," The Journal of Biological Chemistry, vol. 286, no. 46, pp. 40184-40192, 2011.

[44] A. V. Vaseva, N. D. Marchenko, K. Ji, S. E. Tsirka, S. Holzmann, and U. M. Moll, "p53 opens the mitochondrial permeability transition pore to trigger necrosis," Cell, vol. 149, no. 7, pp. 1536-1548, 2012.

[45] G. Gouspillou, N. Sgarioto, S. Kapchinsky et al., "Increased sensitivity to mitochondrial permeability transition and myonuclear translocation of endonuclease $\mathrm{G}$ in atrophied muscle of physically active older humans," The FASEB Journal, vol. 28, no. 4, pp. 1621-1633, 2014.

[46] L. Liu, J. Zhu, P. R. Brink, P. S. Glass, and M. J. Rebecchi, "Age-associated differences in the inhibition of mitochondrial permeability transition pore opening by cyclosporine a," Acta Anaesthesiologica Scandinavica, vol. 55, no. 5, pp. 622-630, 2011.

[47] L. C. Shum, N. S. White, S. M. Nadtochiy et al., "Cyclophilin D Knock-out mice show enhanced resistance to osteoporosis 
and to metabolic changes observed in aging bone," PLoS One, vol. 11, no. 5, article e0155709, 2016.

[48] D. R. Hunter, R. A. Haworth, and J. H. Southard, "Relationship between configuration, function, and permeability in calcium- treated mitochondria.," The Journal of Biological Chemistry, vol. 251, no. 16, pp. 5069-5077, 1976.

[49] S. Hurst, J. Hoek, and S. S. Sheu, "Mitochondrial $\mathrm{Ca}^{2+}$ and regulation of the permeability transition pore," Journal of Bioenergetics and Biomembranes, vol. 49, no. 1, pp. 27-47, 2017.

[50] P. Bernardi, A. Krauskopf, E. Basso et al., "The mitochondrial permeability transition from in vitro artifact to disease target," The FEBS Journal, vol. 273, no. 10, pp. 2077-2099, 2006.

[51] D. F. Dai, Y. Chiao, D. J. Marcinek, H. H. Szeto, and P. S. Rabinovitch, "Mitochondrial oxidative stress in aging and healthspan," Longevity \& healthspan, vol. 3, no. 1, 2014.

[52] M. Forkink, F. Basit, J. Teixeira, H. G. Swarts, W. J. H. Koopman, and P. H. G. M. Willems, "Complex I and complex III inhibition specifically increase cytosolic hydrogen peroxide levels without inducing oxidative stress in HEK293 cells," Redox Biology, vol. 6, pp. 607-616, 2015.

[53] D. B. Zorov, C. R. Filburn, L. O. Klotz, J. L. Zweier, and S. J. Sollott, "Reactive oxygen species (ROS)-induced ROS release: a new phenomenon accompanying induction of the mitochondrial permeability transition in cardiac myocytes," The Journal of Experimental Medicine, vol. 192, no. 7, pp. 10011014, 2000.

[54] F. di Lisa, M. Giorgio, P. Ferdinandy, and R. Schulz, "New aspects of p66Shc in ischaemia reperfusion injury and other cardiovascular diseases," British Journal of Pharmacology, vol. 174, no. 12, pp. 1690-1703, 2017.

[55] C. Priami, G. de Michele, F. Cotelli et al., "Modelling the p53/p66Shc aging pathway in the shortest living vertebrate Nothobranchius furzeri," Aging and Disease, vol. 6, no. 2, pp. 95-108, 2015.

[56] C. Savino, P. Pelicci, and M. Giorgio, "The P66Shc/mitochondrial permeability transition pore pathway determines neurodegeneration," Oxidative Medicine and Cellular Longevity, vol. 2013, Article ID 719407, 7 pages, 2013.

[57] D. L. Steen and M. L. O’Donoghue, "Lp-PLA2 inhibitors for the reduction of cardiovascular events," Cardiology and therapy, vol. 2, no. 2, pp. 125-134, 2013.

[58] A. Riemann, B. Schneider, A. Ihling et al., "Acidic environment leads to ROS-induced MAPK signaling in cancer cells," PLoS One, vol. 6, no. 7, article e22445, 2011.

[59] M. Carraro and P. Bernardi, "Calcium and reactive oxygen species in regulation of the mitochondrial permeability transition and of programmed cell death in yeast," Cell Calcium, vol. 60, no. 2, pp. 102-107, 2016.

[60] M. L. Smith, R. von Hanwehr, and B. K. Siesjö, "Changes in extra- and intracellular $\mathrm{pH}$ in the brain during and following ischemia in hyperglycemic and in moderately hypoglycemic rats," Journal of Cerebral Blood Flow and Metabolism, vol. 6, no. 5, pp. 574-583, 1986.

[61] P. M. Kerr, M. S. Suleiman, and A. P. Halestrap, "Reversal of permeability transition during recovery of hearts from ischemia and its enhancement by pyruvate," The American Journal of Physiology, vol. 276, no. 2, pp. H496-H502, 1999.

[62] S. Javadov, A. Choi, V. Rajapurohitam, A. Zeidan, A. G. Basnakian, and M. Karmazyn, "NHE-1 inhibition-induced cardioprotection against ischaemia/reperfusion is associated with attenuation of the mitochondrial permeability transition," Cardiovascular Research, vol. 77, no. 2, pp. 416-424, 2008.

[63] D. Han, F. Antunes, R. Canali, D. Rettori, and E. Cadenas, "Voltage-dependent anion channels control the release of the superoxide anion from mitochondria to cytosol," The Journal of Biological Chemistry, vol. 278, no. 8, pp. 55575563, 2003.

[64] V. Shoshan-Barmatz, V. de Pinto, M. Zweckstetter, Z. Raviv, N. Keinan, and N. Arbel, "VDAC, a multi-functional mitochondrial protein regulating cell life and death," Molecular Aspects of Medicine, vol. 31, no. 3, pp. 227-285, 2010.

[65] N. Tajeddine, "How do reactive oxygen species and calcium trigger mitochondrial membrane permeabilisation?," Biochimica et Biophysica Acta, vol. 1860, no. 6, pp. 1079-1088, 2016.

[66] A. N. Antony, M. Paillard, C. Moffat et al., "MICU1 regulation of mitochondrial $\mathrm{Ca}^{2+}$ uptake dictates survival and tissue regeneration," Nature Communications, vol. 7, no. 1, article 10955, 2016.

[67] H. Rottenberg and M. Marbach, "Regulation of $\mathrm{Ca}^{2+}$ transport in brain mitochondria. II. The mechanism of the adenine nucleotides enhancement of $\mathrm{Ca}^{2+}$ uptake and retention," Biochimica et Biophysica Acta, vol. 1016, no. 1, pp. 87-98, 1990.

[68] M. P. Mattson, "Calcium and neurodegeneration," Aging Cell, vol. 6, no. 3, pp. 337-350, 2007.

[69] H. Tsai, C. W. Hewitt, J. N. Buchholz, and S. P. Duckles, "Intracellular calcium buffering declines in aging adrenergic nerves," Neurobiology of Aging, vol. 18, no. 2, pp. 229-233, 1997.

[70] C. Fernandez-Sanz, M. Ruiz-Meana, E. Miro-Casas et al., "Defective sarcoplasmic reticulum-mitochondria calcium exchange in aged mouse myocardium," Cell Death \& Disease, vol. 5, no. 12, article e1573, 2014.

[71] D. C. Andersson, M. J. Betzenhauser, S. Reiken et al., "Ryanodine receptor oxidation causes intracellular calcium leak and muscle weakness in aging," Cell Metabolism, vol. 14, no. 2, pp. 196-207, 2011.

[72] L. L. Cooper, W. Li, Y. Lu et al., "Redox modification of ryanodine receptors by mitochondria-derived reactive oxygen species contributes to aberrant $\mathrm{Ca}^{2+}$ handling in ageing rabbit hearts," The Journal of Physiology, vol. 591, no. 23, pp. 58955911, 2013.

[73] M. Calvo-Rodríguez, M. García-Durillo, C. Villalobos, and L. Núñez, "In vitro aging promotes endoplasmic reticulum (ER)-mitochondria $\mathrm{Ca}^{2}+$ cross talk and loss of storeoperated $\mathrm{Ca}^{2}+$ entry (SOCE) in rat hippocampal neurons," Biochimica et Biophysica Acta, vol. 1863, no. 11, pp. 26372649, 2016.

[74] J. C. Liu, J. Liu, K. M. Holmström et al., "MICU1 serves as a molecular gatekeeper to prevent in vivo mitochondrial calcium overload," Cell Reports, vol. 16, no. 6, pp. 1561-1573, 2016.

[75] M. Mather and H. Rottenberg, "Aging enhances the activation of the permeability transition pore in mitochondria," Biochemical and Biophysical Research Communications, vol. 273, no. 2, pp. 603-608, 2000.

[76] B. Golia, H. R. Singh, and G. Timinszky, "Poly-ADP-ribosylation signaling during DNA damage repair," Front Biosci (Landmark Ed), vol. 20, pp. 440-457, 2015. 
[77] P. I. Merksamer, Y. Liu, W. He, M. D. Hirschey, D. Chen, and E. Verdin, "The sirtuins, oxidative stress and aging: an emerging link," Aging (Albany NY), vol. 5, no. 3, pp. 144-150, 2013.

[78] J. Shin, M. Mohrin, and D. Chen, "Reversing stem cell aging," Oncotarget, vol. 6, no. 17, pp. 14723-14724, 2015.

[79] B. H. Ahn, H. S. Kim, S. Song et al., "A role for the mitochondrial deacetylase Sirt3 in regulating energy homeostasis," Proceedings of the National Academy of Sciences of the United States of America, vol. 105, no. 38, pp. 14447-14452, 2008.

[80] M. D. Hirschey, T. Shimazu, E. Goetzman et al., "SIRT3 regulates mitochondrial fatty-acid oxidation by reversible enzyme deacetylation," Nature, vol. 464, no. 7285, pp. 121$125,2010$.

[81] A. P. Gomes, N. L. Price, A. J. Y. Ling et al., "Declining NAD" induces a pseudohypoxic state disrupting nuclearmitochondrial communication during aging," Cell, vol. 155, no. 7, pp. 1624-1638, 2013.

[82] S. Imai and L. Guarente, "NAD ${ }^{+}$and sirtuins in aging and disease," Trends in Cell Biology, vol. 24, no. 8, pp. 464-471, 2014.

[83] J. Q. Kwong and J. D. Molkentin, "Physiological and pathological roles of the mitochondrial permeability transition pore in the heart," Cell Metabolism, vol. 21, no. 2, pp. 206214, 2015.

[84] A. V. Hafner, J. Dai, A. P. Gomes et al., "Regulation of the mPTP by SIRT3-mediated deacetylation of CypD at lysine 166 suppresses age-related cardiac hypertrophy," Aging (Albany NY), vol. 2, no. 12, pp. 914-923, 2010.

[85] G. Barreto-Torres, J. S. Hernandez, S. Jang et al., "The beneficial effects of AMP kinase activation against oxidative stress are associated with prevention of PPAR $\alpha$-cyclophilin D interaction in cardiomyocytes," American Journal of Physiology. Heart and Circulatory Physiology, vol. 308, no. 7, pp. H749H758, 2015.

[86] D. Harman, "Aging: a theory based on free radical and radiation chemistry," Journal of Gerontology, vol. 11, no. 3, pp. 298-300, 1956.

[87] J. Campisi and L. Robert, "Cell senescence: role in aging and age-related diseases," Interdisciplinary Topics in Gerontology, vol. 39, pp. 45-61, 2014.

[88] P. Davalli, T. Mitic, A. Caporali, A. Lauriola, and D. D'Arca, "ROS, cell senescence, and novel molecular mechanisms in aging and age-related diseases," Oxidative Medicine and Cellular Longevity, vol. 2016, Article ID 3565127, 18 pages, 2016.

[89] C. López-Otín, M. A. Blasco, L. Partridge, M. Serrano, and G. Kroemer, "The hallmarks of aging," Cell, vol. 153, no. 6, pp. 1194-1217, 2013.

[90] T. Hofer, S. Servais, A. Y. Seo et al., "Bioenergetics and permeability transition pore opening in heart subsarcolemmal and interfibrillar mitochondria: effects of aging and lifelong calorie restriction," Mechanisms of Ageing and Development, vol. 130, no. 5, pp. 297-307, 2009.

[91] B. Zhou, J. Kreuzer, C. Kumsta et al., "Mitochondrial permeability uncouples elevated autophagy and lifespan extension," Cell, vol. 177, no. 2, pp. 299-314.e16, 2019.

[92] H. Braak and E. Braak, "Pathoanatomy of Parkinson's disease," Journal of Neurology, vol. 247, Supplement 2, pp. II3I10, 2000.

[93] A. M. Graybiel, "The basal ganglia: learning new tricks and loving it," Current Opinion in Neurobiology, vol. 15, no. 6, pp. 638-644, 2005.
[94] M. H. R. Ludtmann, P. R. Angelova, M. H. Horrocks et al., " $\alpha$-synuclein oligomers interact with ATP synthase and open the permeability transition pore in Parkinson's disease," Nature communications, vol. 9, no. 1, 2018.

[95] D. Blum, S. Torch, N. Lambeng et al., "Molecular pathways involved in the neurotoxicity of 6-OHDA, dopamine and MPTP: contribution to the apoptotic theory in Parkinson's disease," Progress in Neurobiology, vol. 65, no. 2, pp. 135$172,2001$.

[96] R. B. Mythri, C. Venkateshappa, G. Harish et al., "Evaluation of markers of oxidative stress, antioxidant function and astrocytic proliferation in the striatum and frontal cortex of Parkinson's disease brains," Neurochemical Research, vol. 36, no. 8, pp. 1452-1463, 2011.

[97] H. Reichmann, "View point: etiology in Parkinson's disease. Dual hit or spreading intoxication," Journal of the Neurological Sciences, vol. 310, no. 1-2, pp. 9-11, 2011.

[98] G. Forloni and C. Balducci, "Alzheimer's disease, oligomers, and inflammation," Journal of Alzheimer's Disease, vol. 62, no. 3, pp. 1261-1276, 2018.

[99] S. Vivekanantham, S. Shah, R. Dewji, A. Dewji, C. Khatri, and R. Ologunde, "Neuroinflammation in Parkinson's disease: role in neurodegeneration and tissue repair," The International Journal of Neuroscience, vol. 125, no. 10, pp. 717-725, 2015.

[100] E. McGowan, F. Pickford, J. Kim et al., “A $\beta 42$ Is Essential for Parenchymal and Vascular Amyloid Deposition in Mice," Neuron, vol. 47, no. 2, pp. 191-199, 2005.

[101] D. J. Selkoe, “Toward a comprehensive theory for Alzheimer's disease. Hypothesis: Alzheimer's disease is caused by the cerebral accumulation and cytotoxicity of amyloid beta-protein," Annals of the New York Academy of Sciences, vol. 924, pp. 17$25,2000$.

[102] J. W. Lustbader, M. Cirilli, C. Lin et al., "ABAD directly links Abeta to mitochondrial toxicity in Alzheimer's disease," Science, vol. 304, no. 5669, pp. 448-452, 2004.

[103] P. H. Reddy and M. F. Beal, "Amyloid beta, mitochondrial dysfunction and synaptic damage: implications for cognitive decline in aging and Alzheimer's disease," Trends in Molecular Medicine, vol. 14, no. 2, pp. 45-53, 2008.

[104] Y. Rui, P. Tiwari, Z. Xie, and J. Q. Zheng, “Acute impairment of mitochondrial trafficking by beta-amyloid peptides in hippocampal neurons," The Journal of Neuroscience, vol. 26, no. 41, pp. 10480-10487, 2006.

[105] K. Takuma, J. Yao, J. Huang et al., "ABAD enhances Abetainduced cell stress via mitochondrial dysfunction," The FASEB Journal, vol. 19, no. 6, pp. 597-598, 2005.

[106] F. Bosetti, F. Brizzi, S. Barogi et al., "Cytochrome c oxidase and mitochondrial $\mathrm{F}_{1} \mathrm{~F}_{0}$-ATPase (ATP synthase) activities in platelets and brain from patients with Alzheimer's disease," Neurobiology of Aging, vol. 23, no. 3, pp. 371-376, 2002.

[107] S. Ohta and I. Ohsawa, "Dysfunction of mitochondria and oxidative stress in the pathogenesis of Alzheimer's disease: on defects in the cytochrome c oxidase complex and aldehyde detoxification," Journal of Alzheimer's Disease, vol. 9, no. 2, pp. 155-166, 2006.

[108] A. M. Aleardi, G. Benard, O. Augereau et al., "Gradual alteration of mitochondrial structure and function by $\beta$-amyloids: importance of membrane viscosity changes, energy deprivation, reactive oxygen species production, and cytochrome $c$ 
release," Journal of Bioenergetics and Biomembranes, vol. 37, no. 4, pp. 207-225, 2005.

[109] H. du, L. Guo, F. Fang et al., "Cyclophilin D deficiency attenuates mitochondrial and neuronal perturbation and ameliorates learning and memory in Alzheimer's disease," Nature Medicine, vol. 14, no. 10, pp. 1097-1105, 2008.

[110] E. Basso, L. Fante, J. Fowlkes, V. Petronilli, M. A. Forte, and P. Bernardi, "Properties of the Permeability Transition Pore in Mitochondria Devoid of Cyclophilin D," The Journal of Biological Chemistry, vol. 280, no. 19, pp. 18558-18561, 2005.

[111] H. du, L. Guo, W. Zhang, M. Rydzewska, and S. Yan, "Cyclophilin D deficiency improves mitochondrial function and learning/memory in aging Alzheimer disease mouse model," Neurobiology of Aging, vol. 32, no. 3, pp. 398-406, 2011.

[112] S. Sanz-Blasco, R. A. Valero, I. Rodríguez-Crespo, C. Villalobos, and L. Núñez, "Mitochondrial $\mathrm{Ca}^{2+}$ overload underlies $\mathrm{A} \beta$ oligomers neurotoxicity providing an unexpected mechanism of neuroprotection by NSAIDs," PLoS One, vol. 3, no. 7, article e2718, 2008.

[113] T. Briston, D. L. Selwood, G. Szabadkai, and M. R. Duchen, "Mitochondrial permeability transition: a molecular lesion with multiple drug targets," Trends in Pharmacological Sciences, vol. 40, no. 1, pp. 50-70, 2019.

[114] P. C. Waldmeier, J. J. Feldtrauer, T. Qian, and J. J. Lemasters, "Inhibition of the mitochondrial permeability transition by the nonimmunosuppressive cyclosporin derivative NIM811," Molecular Pharmacology, vol. 62, no. 1, pp. 22$29,2002$.

[115] N. Fournier, G. Ducet, and A. Crevat, "Action of cyclosporine on mitochondrial calcium fluxes," Journal of Bioenergetics and Biomembranes, vol. 19, no. 3, pp. 297-303, 1987.

[116] M. Crompton, H. Ellinger, and A. Costi, "Inhibition by cyclosporin a of a $\mathrm{Ca}^{2+}$-dependent pore in heart mitochondria activated by inorganic phosphate and oxidative stress," The Biochemical Journal, vol. 255, no. 1, pp. 357-360, 1988.

[117] A. Tanveer, S. Virji, L. Andreeva et al., "Involvement of cyclophilin $\mathrm{D}$ in the activation of a mitochondrial pore by $\mathrm{Ca}^{2+}$ and oxidant stress," European Journal of Biochemistry, vol. 238, no. 1, pp. 166-172, 1996.

[118] E. J. Griffiths and A. P. Halestrap, "Protection by cyclosporin A of ischemia/reperfusion-induced damage in isolated rat hearts," Journal of Molecular and Cellular Cardiology, vol. 25, no. 12, pp. 1461-1469, 1993.

[119] R. M. Parodi-Rullán, J. Soto-Prado, J. Vega-Lugo, X. ChapaDubocq, S. I. Díaz-Cordero, and S. Javadov, "Divergent effects of cyclophilin-D inhibition on the female rat heart: acute versus chronic post-myocardial infarction," Cellular Physiology and Biochemistry, vol. 50, no. 1, pp. 288-303, 2018.

[120] J. E. Springer, N. P. Visavadiya, P. G. Sullivan, and E. D. Hall, "Post-injury treatment with NIM811 promotes recovery of function in adult female rats after spinal cord contusion: a dose-response study," Journal of Neurotrauma, vol. 35, no. 3, pp. 492-499, 2018.

[121] R. D. Readnower, J. D. Pandya, M. L. McEwen, J. R. Pauly, J. E. Springer, and P. G. Sullivan, "Post-injury administration of the mitochondrial permeability transition pore inhibitor, NIM811, is neuroprotective and improves cognition after traumatic brain injury in rats," Journal of Neurotrauma, vol. 28, no. 9, pp. 1845-1853, 2011.
[122] K. B. El Baradie, M. Khan, B. Mendhe, J. Waller, F. O'Brien III, and M. W. Hamrick, "NIM-811, a cyclophilin inhibitor, increases muscle cell survival with hypoxia in vitro and improves measures of gait performance and muscle inflammation following ischemia-reperfusion in vivo," Scientific Reports, vol. 23, no. 20, pp. 5353-5363, 2014.

[123] Y. Baburina, I. Odinokova, T. Azarashvili, V. Akatov, J. J. Lemasters, and O. Krestinina, " 2 ', $3^{\prime}$-Cyclic nucleotide $3^{\prime}$ -phosphodiesterase as a messenger of protection of the mitochondrial function during melatonin treatment in aging," Biochimica et Biophysica Acta - Biomembranes, vol. 1859, no. 1, pp. 94-103, 2017.

[124] S. A. Andrabi, I. Sayeed, D. Siemen, G. Wolf, and T. F. W. Horn, "Direct inhibition of the mitochondrial permeability transition pore: a possible mechanism responsible for antiapoptotic effects of melatonin," The FASEB Journal, vol. 18, no. 7, pp. 869-871, 2004.

[125] R. Y. Liu, J. N. Zhou, J. van Heerikhuize, M. A. Hofman, and D. F. Swaab, "Decreased melatonin levels in postmortem cerebrospinal fluid in relation to aging, Alzheimer's disease, and apolipoprotein E-epsilon4/4 genotype," The Journal of Clinical Endocrinology and Metabolism, vol. 84, no. 1, pp. 323-327, 1999.

[126] A. P. Halestrap, G. P. McStay, and S. J. Clarke, "The permeability transition pore complex: another view," Biochimie, vol. 84, no. 2-3, pp. 153-166, 2002.

[127] D. Fancelli, A. Abate, R. Amici et al., "Cinnamic anilides as new mitochondrial permeability transition pore inhibitors endowed with ischemia-reperfusion injury protective effect in vivo," Journal of Medicinal Chemistry, vol. 57, no. 12, pp. 5333-5347, 2014.

[128] L. J. Martin, D. Fancelli, M. Wong et al., "GNX-4728, a novel small molecule drug inhibitor of mitochondrial permeability transition, is therapeutic in a mouse model of amyotrophic lateral sclerosis," Frontiers in Cellular Neuroscience, vol. 8, p. 433, 2014.

[129] S. Roy, J. Šileikyte, B. Neuenswander et al., "N-Phenylbenzamides as potent inhibitors of the mitochondrial permeability transition pore," ChemMedChem, vol. 11, no. 3, pp. 283-288, 2016.

[130] S. Roy, J. Šileikyte, M. Schiavone et al., "Discovery, synthesis, and optimization of diarylisoxazole-3-carboxamides as potent inhibitors of the mitochondrial permeability transition pore," ChemMedChem, vol. 10, no. 10, pp. 1655-1671, 2015.

[131] K. Zhao, G. M. Zhao, D. Wu et al., "Cell-permeable Peptide Antioxidants Targeted to Inner Mitochondrial Membrane inhibit Mitochondrial Swelling, Oxidative Cell Death, and Reperfusion Injury," The Journal of Biological Chemistry, vol. 279, no. 33, pp. 34682-34690, 2004.

[132] N. Escobales, R. E. Nuñez, S. Jang et al., "Mitochondria-targeted ROS scavenger improves post-ischemic recovery of cardiac function and attenuates mitochondrial abnormalities in aged rats," Journal of Molecular and Cellular Cardiology, vol. 77, pp. 136-146, 2014.

[133] J. Ji, A. E. Kline, A. Amoscato et al., "Lipidomics identifies cardiolipin oxidation as a mitochondrial target for redox therapy of brain injury," Nature Neuroscience, vol. 15, no. 10, pp. 1407-1413, 2012.

[134] V. J. Adlam, J. C. Harrison, C. M. Porteous et al., "Targeting an antioxidant to mitochondria decreases cardiac ischemia- 
reperfusion injury," The FASEB Journal, vol. 19, no. 9, pp. 1088-1095, 2005.

[135] S. Kawakami, A. Matsuda, T. Sunagawa et al., "Antioxidant, EUK-8, prevents murine dilated cardiomyopathy," Circulation Journal, vol. 73, no. 11, pp. 2125-2134, 2009.

[136] H. Hu and M. Li, "Mitochondria-targeted antioxidant mitotempo protects mitochondrial function against amyloid beta toxicity in primary cultured mouse neurons," Biochemical and Biophysical Research Communications, vol. 478, no. 1, pp. 174-180, 2016.

[137] S. Matsumoto, M. Murozono, M. Kanazawa, T. Nara, T. Ozawa, and Y. Watanabe, "Edaravone and cyclosporine a as neuroprotective agents for acute ischemic stroke," Acute medicine \& surgery, vol. 5, no. 3, pp. 213-221, 2018.

[138] A. P. Halestrap, "The C ring of the F1Fo ATP synthase forms the mitochondrial permeability transition pore: a critical appraisal," Frontiers in Oncology, vol. 4, 2014.

[139] W. Nazareth, N. Yafei, and M. Crompton, "Inhibition of anoxia-induced injury in heart myocytes by cyclosporin a," Journal of Molecular and Cellular Cardiology, vol. 23, no. 12, pp. 1351-1354, 1991.

[140] Y. Takayasu, J. Nakaki, T. Kawasaki et al., "Edaravone, a radical scavenger, inhibits mitochondrial permeability transition pore in rat brain," Journal of Pharmacological Sciences, vol. 103, no. 4, pp. 434-437, 2007.

[141] J. Wu, M. Zhang, H. Li et al., "BDNF pathway is involved in the protective effects of SS-31 on isoflurane- induced cognitive deficits in aging mice," Behavioural Brain Research, vol. 305, pp. 115-121, 2016.

[142] S. Javadov, S. Jang, N. Rodriguez-Reyes et al., "Mitochondriatargeted antioxidant preserves contractile properties and mitochondrial function of skeletal muscle in aged rats," Oncotarget, vol. 6, no. 37, pp. 39469-39481, 2015.

[143] V. P. van Empel, A. T. Bertrand, R. J. van Oort et al., "EUK-8, a superoxide dismutase and catalase mimetic, reduces cardiac oxidative stress and ameliorates pressure overload-induced heart failure in the harlequin mouse mutant," Journal of the American College of Cardiology, vol. 48, no. 4, pp. 824-832, 2006. 